\title{
Influencia del contenido metálico sobre las constantes ópticas de laminas delgadas amorfas de composición $\mathrm{As}_{33} \mathrm{~S}_{67}$ fotodopadas con $\mathrm{Ag}$
}

\author{
J.M. GONZÁLEZ-LEAL, T. WAGNER', A.M. BERNAL-OLIVA, R. PRIETO-ALCÓN Y E. MÁRQUEZ
}

Departamento de Física de la Materia Condensada. Facultad de Ciencias. Universidad de Cádiz. 11510 - Puerto Real (Cádiz). ${ }^{1}$ Department of General and Inorganic Chemistry. Faculty of Chemical Technology. University of Pardubice. 53210 - Pardubice. Czech Republic.

\begin{abstract}
Se han preparado láminas delgadas amorfas calcogenuras de composición $\operatorname{Ag}_{\mathrm{x}}\left(\mathrm{As}_{0.33} \mathrm{~S}_{0.67}\right)_{100-x^{\prime}}$ mediante el fenómeno fotoinducido conocido como fotodisolución o fotodopaje, alcanzándose concentraciones de Ag en las muestras de hasta un $16.5 \%$ at. Las constantes ópticas de las láminas fotodopadas, se han determinado haciendo uso de un método de caracterización basado en las envolventes de los espectros de transmisión óptica obtenidos en incidencia normal. El análisis de la dispersión del índice de refracción se ha realizado siguiendo el modelo propuesto por Wemple-DiDomenico, basado en la aproximación del oscilador armónico simple. Este modelo ha permitido obtener información estructural de las láminas fotodopadas, a partir de los resultados ópticos. El borde de absorción óptica de las láminas fotodopadas se ha analizado sobre la base del modelo de transición no directa propuesto por Tauc, encontrando una disminución del gap óptico del producto fotodopado, con el contenido de plata.
\end{abstract}

Palabras clave: semiconductores amorfos, vidrios calcogenuros, láminas delgadas, constantes ópticas, efectos fotoinducidos.

Influence of the metal content in the optical constants of silver-photodoped $\mathrm{As}_{33} \mathrm{~S}_{67}$ amorphous thin films

$\mathrm{Ag}_{\mathrm{x}}\left(\mathrm{As}_{0.33} \mathrm{~S}_{0.67}\right)_{100-\mathrm{x}}$ amorphous chalcogenide thin films have been prepared by the process of photodissolution or photodoping, reaching silver concentrations of up to 16.5 at. \%. The optical constants of the photodoped thin-film samples have been calculated from the envelopes of the transmission spectra, taken at normal incidence. The dispersion of the refractive index was analysed on the basis of the single-oscillator model proposed by Wemple and DiDomenico. This model allows obtaining structural information of the silver-photodoped layers from optical data. The optical absorption edge is described using the non-direct transition model suggested by Tauc, and a clear decrease of the optical gap of the photodoped product, with the silver content, has been found.

Keywords: amorphous semiconductors, chalcogenide glasses, thin films, optical constants, photoinduced effects.

\section{INTRODUCCION.}

Los materiales calcogenuros amorfos resultan de gran interés dada la variedad de efectos fotoinducidos que muestran. Entre ellos, podemos citar los siguientes: fotovaporización, fotopolimerización, fotovitrificación y fotodisolución de ciertos metales (habitualmente Ag y Zn) (1-3). Todos ellos dan lugar a cambios en las constantes ópticas, así como a desplazamientos del borde de absorción (fotooscurecimiento o fotoaclarado) de estos materiales (4). El más atractivo de cara a sus posibles aplicaciones tecnológicas en el campo del registro óptico y en la fabricación de dispositivos ópticos difractivos es, sin duda, el fenómeno de la fotodisolución de metales, también denominado fotodopaje $(1,5)$. Este fenómeno consiste en la incorporación de iones metálicos en el seno del material calcogenuro, mediante la exposición a radiación de energía cercana al valor del gap óptico del material (usualmente luz ultravioleta) $(1,6)$. Aunque el mecanismo de este proceso no está aún suficientemente esclarecido, algunos autores sugieren que se trata de una reacción de intercalación en estado sólido, estimulada por la iluminación $(7,8)$.

El objetivo de este trabajo consiste en la determinación de los cambios inducidos en las constantes ópticas, índice de refracción $n(\lambda)$ y coeficiente de extinción $k(\lambda)$, de láminas delgadas de composición química $\mathrm{As}_{33} \mathrm{~S}_{67}$, preparadas mediante deposición de vapor, como consecuencia de la incorporación sucesiva de plata al material calcogenuro, mediante el proceso de fotodisolución de metales. La caracterización óptica de las láminas fotodopadas, de composición química $\operatorname{Ag}_{x}\left(\mathrm{As}_{0.33} \mathrm{~S}_{0.67}\right)_{100-x}(x \leq 16.5 \%$ at. $)$, se ha llevado a cabo aplicando un método basado en las envolventes del espectro de transmisión óptica obtenido en incidencia normal (9), el cual permite además calcular el espesor de éstas.

\section{PROCEDIMIENTO EXPERIMENTAL.}

Las láminas delgadas de composición química $\mathrm{As}_{33} \mathrm{~S}_{67}$ fueron preparadas usando la técnica de deposición de vapor en vacío. Esta composición es la más favorable dentro del sistema vítreo As-S al fenómeno de la fotodisolución de metales y la que garantiza además una excelente homogeneidad del producto fotodopado (10). El material amorfo de partida para la preparación de las láminas se sintetizó en forma de lingote a partir de los elementos constituyentes, usando la conocida técnica del enfriamiento brusco de la mezcla fundida ("meltquenching"). El proceso de evaporación térmica fue llevado a cabo, a partir de fragmentos del lingote vítreo, en un evapora- 
dor (Tesla Corporation, modelo UP-858), a una presión de $\approx 10^{-4} \mathrm{~Pa}$. La velocidad de deposición se fijó en $\approx 1 \mathrm{~nm} / \mathrm{s}$. Durante la deposición, los substratos se mantuvieron girando mediante un sistema denominado de rotación planetaria, lo que hizo posible obtener un excelente grado de paralelismo entre las caras de las láminas así preparadas. Se llevaron a cabo varias deposiciones en las mismas condiciones anteriores, controlando los espesores finales de las láminas depositadas dentro del intervalo comprendido entre 800 y $1200 \mathrm{~nm}$. Posteriormente, sobre las láminas calcogenuras se depositaron y fotodisolvieron de forma consecutiva, láminas de plata de diferentes espesores. Para la evaporación de la plata se usó un crisol de volframio con un recubrimiento de $\mathrm{Al}_{2} \mathrm{O}_{3}$, para reducir la exposición de las láminas al calor y a la luz durante la deposición, a fin de evitar posibles efectos termo- y fotoinducidos no controlados. El proceso de fotodopaje se llevó a cabo iluminando las muestras con una lámpara de $\mathrm{Hg}$ de $200 \mathrm{~W}$ de potencia. Durante la exposición a la luz, las muestras se dispusieron entre dos substratos vítreos para evitar posibles fenómenos de fotooxidación y de fotohidrólisis, en la superficie de las láminas calcogenuras (11-13).

Para conseguir muestras con un alto contenido de Ag es aconsejable, en la práctica, realizar un dopado gradual, es decir, fotodisolviendo pequeñas cantidades de plata "paso a paso", en vez de fotodisolver la totalidad de la plata en "un único paso". De esta manera, se consiguen láminas fotodopadas más homogéneas y de mejor calidad óptica. En el caso representativo que se presenta en este trabajo, los espesores de las láminas de plata fotodisueltas en una lámina vítrea calcogenura de composición $\mathrm{As}_{33} \mathrm{~S}_{67}$ y de $960 \mathrm{~nm}$ de espesor, fueron, sucesivamente: 20, 40, 40 y 20 nm; siendo los espesores de Ag acumulados, por tanto: 20, 60, 100 y $120 \mathrm{~nm}$. Los valores de la concentración de plata fueron determinados a partir de las medidas de los espesores de las láminas de plata y del material calcogenuro, con una exactitud de $\pm 2 \%$ at. Así, las concentraciones de plata conseguidas en las presentes muestras fueron: 3.0, 8.6, 13.6 y $16.5 \%$ at. Análisis realizados mediante espectroscopía de masas de los iones secundarios (SIMS), han confirmado que la plata se distribuye uniformemente en las láminas de As-S fotodopadas. Sin embargo, es ciertamente difícil utilizar esta técnica para la determinación precisa del contenido de plata, pues la propia medida afecta a la distribución de plata en las muestras.

Finalmente, los espectros de transmisión óptica en incidencia normal, se han obtenido en el intervalo espectral comprendido entre 400 y $2500 \mathrm{~nm}$, mediante un espectrofotómetro UV / Vis/NIR (ultravioleta-visible-infrarrojo cercano) de doble haz (Perkin Elmer, modelo Lambda-9). Todas las medidas efectuadas en este estudio se han llevado a cabo a temperatura ambiente.

\section{FUNDAMENTOS TEORICOS.}

El sistema óptico en estudio consiste en una lámina delgada de caras planoparalelas, isótropa y débilmente absorbente, depositada sobre un substrato transparente de índice de refracción $s$. El sistema se encuentra rodeado de aire cuyo índice de refracción es $n_{\text {aire }}=1$. La lámina tiene un índice de refracción complejo $n_{\mathrm{c}}=n-\mathrm{i} k$, donde el coeficiente de extinción, $k$,

TABLA I. EJEMPLO REPRESENTATIVO DE LA APLICACIÓN DEL ALGORITMO PARA EL CÁLCULO DEL ESPESOR Y DEL ÍNDICE DE REFRACCIÓN, CORRESPONDIENTE A LA MUESTRA FOTO DOPADA CON MAYOR CONCENTRACIÓN DE Ag, CUYA COMPOSICIÓN QUíMICA ES Ag ${ }_{16.5}$ As $_{27.6} S_{55.9}$ LOS VALORES FINALES DEL ÍNDICE DE REFRACCIÓN DE LA MUESTRA APARECEN RECOGIDOS EN LA COLUMNA $n_{1}$. ASIMISMO, EL ESPESOR SE DETERMINÓ COMO EL PROMEDIO DE AQUELLOS VALORES LISTADOS EN LA COLUMNA $d_{1}$. EL SIGNIFICADO DE TODOS LOS PARÁMETROS QUE APARECEN EN LA TABLA, ESTÁ EXPLICADO CON TODO DETALLE EN OTROS TRABAJOS DE ESTOS MISMOS AUTORES $(15,16)$

\begin{tabular}{|c|c|c|c|c|c|c|c|c|c|}
\hline$s$ & $\lambda(\mathrm{nm})$ & $T_{\mathrm{M}}$ & $T_{\mathrm{m}}$ & $n_{0}$ & $d_{0}(\mathrm{~nm})$ & $m_{0}$ & $m_{1}$ & $d_{1}(\mathrm{~nm})$ & $n_{1}$ \\
\hline 1.485 & 1844 & 0.926 & 0.563 & 2.652 & $\longrightarrow$ & 3.27 & 3.0 & 1043 & 2.634 \\
\hline 1.488 & 1386 & 0.922 & 0.558 & 2.666 & 1049 & 4.37 & 4.0 & 1040 & 2.640 \\
\hline 1.490 & 1245 & 0.924 & 0.557 & 2.677 & 1103 & 4.88 & 4.5 & 1047 & 2.668 \\
\hline 1.488 & 1122 & 0.925 & 0.553 & 2.692 & 1002 & 5.45 & 5.0 & 1042 & 2.671 \\
\hline 1.494 & 1028 & 0.924 & 0.548 & 2.714 & 1040 & 6.00 & 5.5 & 1042 & 2.692 \\
\hline 1.498 & 947 & 0.922 & 0.545 & 2.726 & 1049 & 6.54 & 6.0 & 1042 & 2.706 \\
\hline 1.504 & 889 & 0.919 & 0.542 & 2.741 & 1222 & 7.00 & 6.5 & 1054 & 2.752 \\
\hline 1.505 & 825 & 0.915 & 0.536 & 2.760 & 951 & 7.60 & 7.0 & 1046 & 2.750 \\
\hline 1.506 & 775 & 0.908 & 0.529 & 2.780 & 1032 & 8.15 & 7.5 & 1045 & 2.768 \\
\hline 1.506 & 741 & 0.897 & 0.522 & 2.795 & 1359 & 8.57 & 8.0 & 1061 & 2.823 \\
\hline 1.506 & 701 & 0.882 & 0.511 & 2.818 & 1008 & 9.13 & 8.5 & 1057 & 2.837 \\
\hline 1.507 & 648 & 0.819 & 0.469 & 2.913 & 1134 & 10.2 & 9.5 & 1057 & 2.931 \\
\hline 1.507 & 619 & 0.760 & 0.439 & 2.971 & 819 & 10.9 & 10.0 & 1042 & 2.948 \\
\hline 1.508 & 608 & 0.675 & 0.399 & 3.033 & 1320 & 11.3 & 10.5 & 1052 & 3.040 \\
\hline 1.508 & 593 & 0.578 & 0.357 & 3.085 & 1176 & 11.8 & 11.0 & 1057 & 3.106 \\
\hline 1.509 & 579 & 0.475 & 0.315 & 3.082 & 2052 & 12.1 & 11.5 & 1080 & 3.171 \\
\hline 1.510 & 559 & 0.266 & 0.240 & 2.304 & - & 9.37 & 12.0 & - & 3.194 \\
\hline
\end{tabular}

$\overline{\mathrm{d}}_{0}=1136 \pm 271 \mathrm{~nm}(23.9 \%)$

$\overline{\mathrm{d}_{1}}=1050 \pm 10 \mathrm{~nm}(0.95 \%)$ 
se relaciona con el coeficiente de absorción, $\alpha$, mediante la expresión $k=\alpha \lambda / 4 \pi$. Además, el espesor del substrato es varios órdenes de magnitud superior al espesor de la lámina, d. Bajo estas consideraciones, la transmitancia de este sistema bicapa vendría dada por la siguiente expresión [9]:

$$
T(\lambda)=\frac{A x}{B-C x \cos \varphi+D x^{2}}
$$

donde $A=16 n^{2} s, B=(n+1)^{3}\left(n+s^{2}\right), C=2\left(n^{2}-1\right)\left(n-s^{2}\right), D=$ $(n-1)^{3}\left(n-s^{2}\right), \varphi=4 \pi n d / \lambda$ y $x=\exp (-\alpha d)$. Asimismo, haciendo $\cos \varphi= \pm 1$ en la Ec. (1), se obtendrían las expresiones correspondientes a las envolventes superior, $T_{M}(\lambda)$, e inferior, $T_{m}(\lambda)$, del espectro de transmisión:

$$
T(\lambda)=\frac{A x}{B \pm C x+D x^{2}}
$$

Es sencillo comprobar que de la expresión $\cos \varphi= \pm 1$, se obtendría la conocida ecuación básica para las interferencias:

$$
2 n d=m \lambda,
$$

donde $m$ es un número de orden. Sin embargo, debido a la absorción óptica, esta ecuación se verificará para aquellas longitudes de onda, $\lambda_{\tan }$ en las que se presentan puntos de tangencia entre las envolventes y el espectro de transmisión, y no

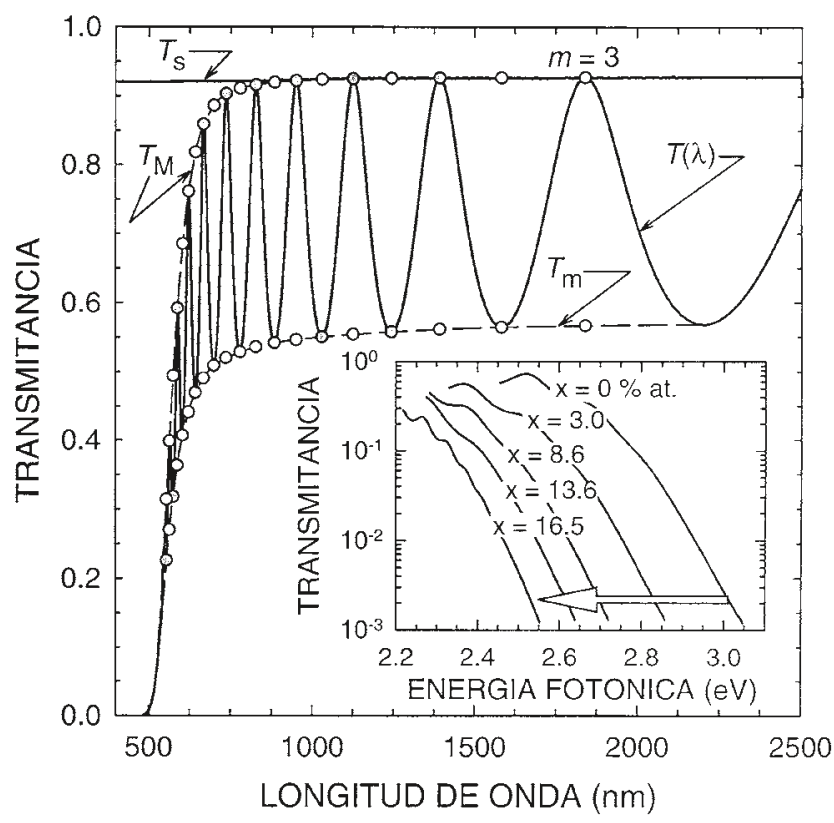

Figura 1. Espectros de transmisión óptica obtenidos en incidencia normal, correspondientes a una lámina fotodopada de composición $\mathrm{Ag}_{16.5} \mathrm{As}_{27.6} \mathrm{~S}_{55.9^{\prime}} T(\lambda)$, y al substrato sobre el que está depositada, $T_{\mathrm{s}}$. Las envolventes superior e inferior, $T_{\mathrm{M}}$ y $T_{\mathrm{m}^{\prime}}$, respectivamente, fueron generadas computacionalmente haciendo uso del algoritmo desarrollado por McClain et al. (18). Se han marcado los puntos de ambas envolventes, para aquellas longitudes de onda en las que éstas son tangentes al espectro. Se indica, además, el número de orden, $m$, correspondiente a un punto de tangencia representativo. En la figura interior se representa, en escala logarítmica y como función de la energía del fotón, la transmisión de las distintas láminas fotodopadas, en la región espectral libre de interferencias. necesariamente en los extremos interferenciales, es decir, aquellos puntos donde $d T(\lambda) / d \lambda=0$ (14). Por otra parte, los valores del índice de refracción del substrato pueden calcularse en todo el rango espectral en estudio, a partir del espectro de transmisión del substrato en solitario, $T_{\mathrm{s}^{\prime}}$ utilizando la siguiente expresión (9):

$$
s=\frac{1}{T_{\mathrm{S}}}+\left(\frac{1}{T_{\mathrm{S}}^{2}}-1\right)^{1 / 2}
$$

Así pues, conocido el índice de refracción del substrato, las ecuaciones incluidas en (2) constituyen un sistema de ecuaciones que puede ser resuelto analíticamente para $n$ y $x$. El índice de refracción de la lámina puede ser entonces calculado en el intervalo espectral cubierto, mediante la siguiente expresión:

$$
n(\lambda)=\sqrt{N+\sqrt{N^{2}-s^{2}}}
$$

donde

$$
N=2 s \frac{T_{\mathrm{M}}(\lambda)-T_{\mathrm{m}}(\lambda)}{T_{\mathrm{M}}(\lambda) T_{\mathrm{m}}(\lambda)}+\frac{s^{2}+1}{2}
$$

Sin embargo, en la práctica, los valores del índice de refracción así calculados, son extraordinariamente sensibles a los errores cometidos en la obtención del espectro de transmisión, así como a los cometidos en el proceso de generación de las envolventes. Por ello, resulta útil limitar el cálculo a aquellas longitudes de onda, $\lambda_{\tan \text { ' }}$ en las que se presentan puntos de tangencia entre las envolventes y el espectro. De esta forma, la

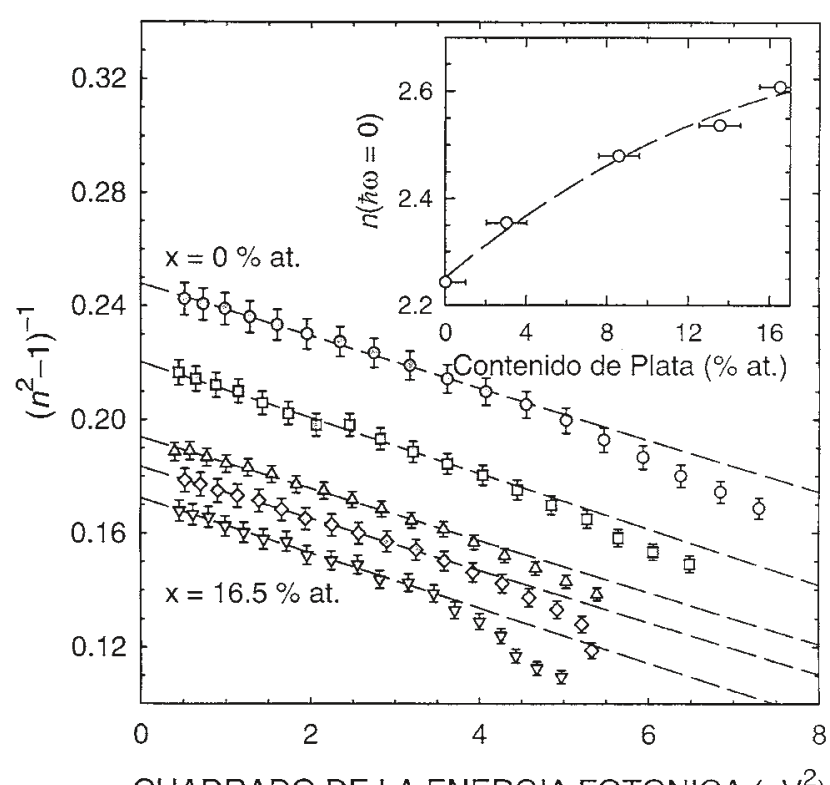

Figura 2. Representación lineal de la expresión del modelo de Wemple-DiDomenico, para el análisis de la dispersión del índice de refracción. Los valores que se alejan del comportamiento esperado según la Ec. (8), aparecen con símbolos en blanco. También se han representado en función del contenido de $\mathrm{Ag}$, los valores del índice de refracción estático, $n(\hbar \omega=0)$, determinados a partir de las ordenadas en el origen de las distintas rectas de regresión. 
Ec. [3] es entonces aplicable, siendo $m$ un número entero para los puntos de tangencia del espectro con la envolvente superior y un número semientero para aquellos con la envolvente inferior. La Ec. [3] permite determinar, además, el correspondiente espesor de la lámina, $d$. Todos los detalles sobre la aplicación práctica del algoritmo utilizado para el cálculo del índice de refracción y del espesor de las muestras en estudio, pueden encontrarse detalladamente explicados en anteriores publicaciones de los autores $(15,16)$. Asimismo, en la tabla I se recogen los resultados de la aplicación de este algoritmo, para la muestra dopada de composición química $\mathrm{Ag}_{16.5} \mathrm{As}_{27.6} \mathrm{~S}_{55.9}$.

Por otra parte, la absorbancia, $x$, puede ser calculada a partir de la envolvente superior, $T_{\mathrm{M}}(\lambda)$, mediante la expresión (9):

$$
x=\frac{E_{\mathrm{M}}-\left[E_{\mathrm{M}}^{2}-\left(n^{2}-1\right)^{3}\left(n^{2}-s^{4}\right)\right]^{1 / 2}}{(n-1)^{3}\left(n-s^{2}\right)}
$$

donde

$$
E_{\mathrm{M}}=\frac{8 n^{2} s}{T_{\mathrm{M}}(\lambda)}+\left(n^{2}-1\right)\left(n^{2}-s^{2}\right)
$$

Conocidos los valores de la absorbancia, $x$, y el espesor de la lámina, $d$, la ecuación $x=\exp (-\alpha d)$ puede entonces resolverse para obtener los valores del coeficiente de absorción, $\alpha$. Finalmente, de la relación entre $k$ y $\alpha$ mencionada anteriormente, puede calcularse el coeficiente de extinción.

Un parámetro de gran importancia desde el punto de vista del estudio de las propiedades ópticas de un semiconductor, es el denominado gap óptico, $E_{\mathrm{g}}$ opt, el cual representa, en el caso de un semiconductor amorfo, la diferencia energética entre los bordes de movilidad de las bandas de valencia y conducción. Este parámetro puede ser determinado a partir del análisis de $\alpha$, en la región de fuerte absorción $\left(\alpha>\approx 10^{4} \mathrm{~cm}^{-1}\right)$, sobre la base del modelo de transición no directa propuesto por Tauc (17) para semiconductores amorfos. En esta región, $\alpha(h \omega)$ obedece

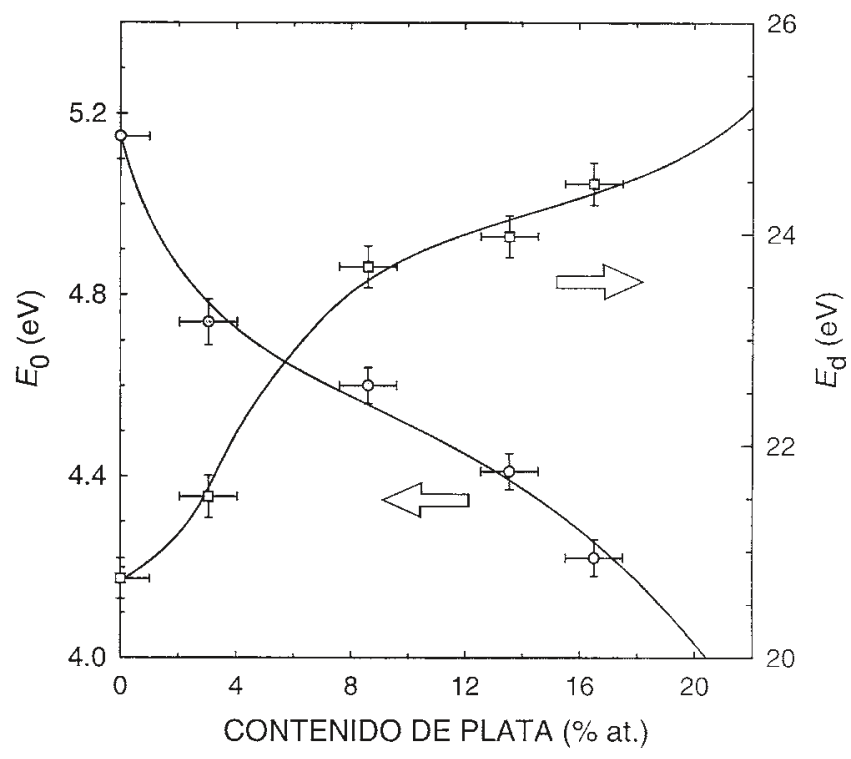

Figura 3. Dependencia de los parámetros de dispersión, $E_{0}$ y $E_{\mathrm{d}^{\prime}}$ del modelo de Wemple-DiDomenico, con el contenido de plata. Las líneas representadas pretenden servir de guía para dar una idea del comportamiento de ambos parámetros. la siguiēnte expresión:

$$
\alpha(\hbar \omega)=\frac{B\left(\hbar \omega-E_{\mathrm{g}}^{\mathrm{opt}}\right)^{2}}{\hbar \omega}
$$

donde $h \omega, E_{\mathrm{g}}{ }^{\text {opt }}$ y $B$ representan, respectivamente, la energía de la radiación, el gap óptico y una constante que depende de la probabilidad de las transiciones electrónicas. Del ajuste lineal de $(\alpha h \omega)^{1 / 2}$ frente a $h \omega$ (Ec. [9]), se obtiene $E_{\mathrm{g}}^{\text {opt }}$ como la intersección con el eje de abscisas.

\section{RESULTADOS Y DISCUSION.}

En la Fig. 1 se muestra el espectro de transmisión óptica, correspondiente a una lámina fotodopada representativa de composición química $\mathrm{Ag}_{16.5} \mathrm{As}_{27.6} \mathrm{~S}_{55.9}$ junto con el espectro del substrato sobre el que se ha depositado, $T_{\mathrm{s}}$. En dicha figura pueden observarse además las envolventes superior e inferior del espectro de transmisión, las cuales se han generado computacionalmente utilizando un algoritmo diseñado por McClain et al. (18) para este propósito. Asimismo, se han marcado los puntos de las envolventes, para aquellas longitudes de onda en las que éstas y el espectro de transmisión son tangentes. En esta misma figura puede verse además, la evolución con el contenido de plata, de los distintos espectros de transmisión de las láminas de composición química $\operatorname{Ag}_{\mathrm{x}}\left(\mathrm{As}_{0.33} \mathrm{~S}_{0.67}\right)_{100-\mathrm{x}}(\mathrm{x}=0$, 3.0, 8.6, 13.6 y $16.5 \%$ at. $)$, obtenidas mediante el proceso de fotodopaje, en la zona espectral correspondiente a bajas longitudes de onda o, equivalentemente, a altas energías. Se observa un claro desplazamiento con el contenido de Ag, de la zona libre de interferencias de estos espectros hacia energías menores, lo que indica un aumento progresivo de la absorción del material fotodopado en la región espectral representada.

Los espesores de las láminas fotodopadas con $\mathrm{Ag}$, determinados a partir de la aplicación del presente método de caracterización óptica, revelaron que el espesor final de la lámina una vez alcanzada la mayor concentración de $\mathrm{Ag}, d\left(\mathrm{Ag}_{16.5} \mathrm{As}_{27.6} \mathrm{~S}_{55.9}\right)$ $=1050 \mathrm{~nm}$, era claramente menor que la suma del espesor de la lámina no dopada inicial, $d\left(\mathrm{As}_{33} \mathrm{~S}_{67}\right)=960 \mathrm{~nm}$, y el espesor acumulado de plata, $d(\mathrm{Ag})=120 \mathrm{~nm}: d\left(\mathrm{As}_{33} \mathrm{~S}_{67}\right)+d(\mathrm{Ag})=1080$ $\mathrm{nm}$. La diferencia encontrada fue de $-30 \mathrm{~nm}$, es decir, una contracción relativa de aproximadamente un $3 \%$. Esta disminución del espesor del producto fotodopado ha sido también encontrada por Kawaguchi et al. (19), en láminas vítreas calcogenuras de las composiciones binarias $\mathrm{As}_{40} \mathrm{~S}_{60}$ y $\mathrm{Ge}_{30} \mathrm{~S}_{70}$. Por otra parte, los espesores de las láminas fotodopadas fueron medidos mecánicamente haciendo uso de un perfilómetro (Sloan, modelo Dektak 3030), presentando en todos los casos, diferencias menores de un $2 \%$ con los valores determinados ópticamente.

El análisis de la dispersión del índice de refracción de las láminas vítreas calcogenuras decomposición $\mathrm{Ag}_{\mathrm{x}}\left(\mathrm{As}_{0.33} \mathrm{~S}_{0.67}\right)_{100-\mathrm{x}}$ $(x=0,3.0,8.6,13.6$ y $16.5 \%$ at. $)$, se ha llevado a cabo haciendo uso del modelo de Wemple-DiDomenico $(20,21)$, basado en la aproximación del oscilador armónico simple:

$$
n^{2}(\hbar \omega)=1+\frac{E_{\mathrm{d}} E_{0}}{E_{0}^{2}-(\hbar \omega)^{2}}
$$

donde $E_{0}$ es la energía del oscilador y $E_{\mathrm{d}}$ es la energía de dispersión. Así, representando $\left(n^{2}-1\right)^{-1}$ frente a $(h \omega)^{2}$ y realizando 
un ajuste lineal, $E_{0}$ y $E_{\mathrm{d}}$ se pueden determinar directamente de la pendiente, $\left(E_{0} E_{\mathrm{d}}\right)^{-1}, \mathrm{y}$ de la ordenada en el origen, $E_{0} / E_{\mathrm{d}}$. En la Fig. 2, se muestran estas representaciones para los valores de $n$ determinados usando el presente método de caracterización óptica, correspondientes a las distintas muestras representativas analizadas en este trabajo. En todas las representaciones se observa que la variación experimental del índice de refracción se aleja del comportamiento espērado según la Ec. (8) a medida que la energía se aproxima $E_{\mathrm{g}}{ }^{\text {opt }} a$, por lo que el modelo de dispersión óptica considerado es sólo válido en las regiones de transparencia y débil absorción. En el recuadro de la Fig. 2, se muestra también la dependencia del índice de refracción estático, $n(h \omega=0)$, con el contenido de plata.

Las dependencias de los parámetros de dispersión, $E_{0} \mathrm{y} E_{\mathrm{d}^{\prime}}$ con la concentración de plata, aparecen representadas en la Fig. 3. A partir de esta figura, se observa un aumento del valor de $E_{\mathrm{d}}$ con el contenido de plata, mientras que el parámetro $E_{0}$ disminuye. Uno de los resultados más atractivos del modelo de dispersión óptica propuesto por Wemple-DiDomenico es la relación empírica encontrada entre el parámetro $E_{\mathrm{d}} \mathrm{y}$ ciertas características de la estructura y el tipo de enlace del material, a través de la siguiente fórmula:

$$
E_{\mathrm{d}}=\beta N_{\mathrm{c}} Z_{\mathrm{a}} N_{\mathrm{e}}
$$

donde $\beta$ es una constante que, para materiales covalentes, tanto amorfos como cristalinos, tiene un valor de $\beta_{c}=0.37 \pm$ $0.04 \mathrm{eV}$ (para materiales iónicos, $\beta_{\mathrm{i}}=0.26 \pm 0.03 \mathrm{eV}$ ), $N_{\mathrm{c}}$ es el número de coordinación del catión, $Z_{\mathrm{a}}$ es la valencia química formal del anión y $N_{\mathrm{e}}$ es el número efectivo de electrones de valencia por anión. Considerando que en el caso de $\mathrm{As}_{40} \mathrm{~S}_{60}$

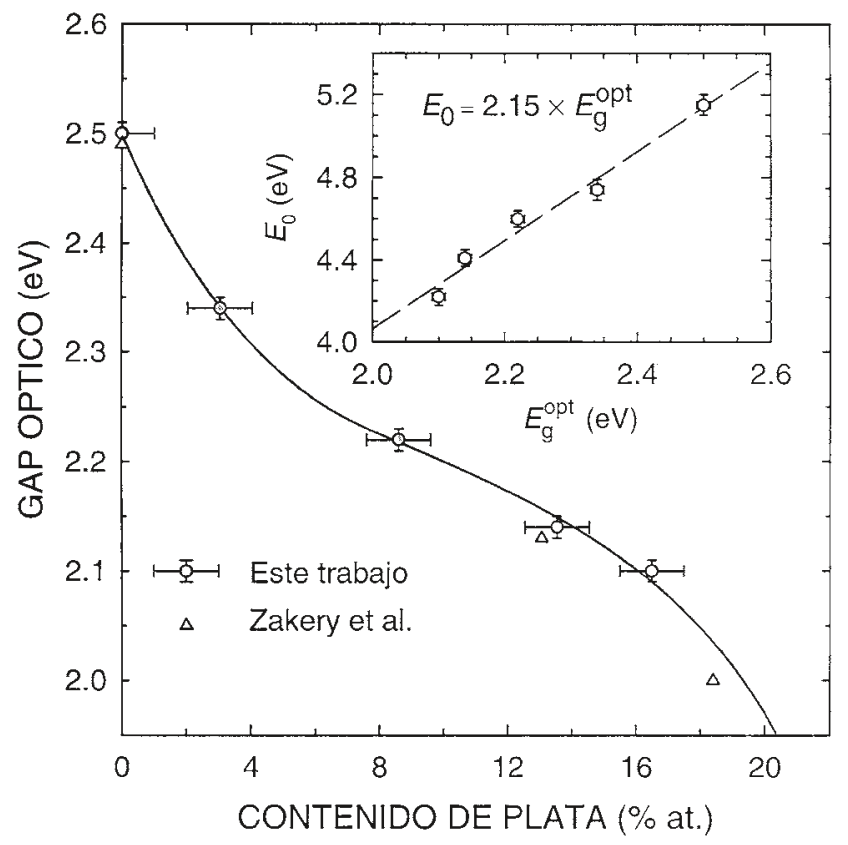

Figura 4. Representación en función del contenido de Ag, de los valores del gap de Tauc, $E_{\mathrm{g}}$ opt, determinados a partir del coeficiente de absorción, en la región correspondiente a valores de $\alpha>\approx 10^{4} \mathrm{~cm}^{-1}$. El acuerdo entre estos valores y aquellos encontrados por Zakery et al. (25), para láminas fotodopadas de composición $\operatorname{Ag}_{\mathrm{x}}\left(\mathrm{As}_{0.30} \mathrm{~S}_{0.70}\right)_{100-\mathrm{x}}(\mathrm{x}=0,13.1 \mathrm{y}$ $18.4 \%$ at.), es ciertamente notable. La representación del parámetro de dispersión $E_{0}$ frente a $E_{\mathrm{g}}{ }^{\mathrm{ppt}}$, en el recuadro de la figura pone de manifiesto la clara proporcionalidad entre ambos parámetros ópticos. cristalino, algunos de los átomos de As actúan uniendo entre sí las capas bi-dimensionales alabeadas de As-S que presenta la estructura, se puede argumentar que estos átomos contribuyen a incrementar el número de coordinación medio del As y, sería necesario tomar un valor de $N_{c} \approx 3.6$, con el fin de obtener el valor covalente del parámetro $\beta$. Sin embargo, esta contribución es de esperar que sea menor en la fase amorfa, con lo que el número de coordinación medio del As disminuiría, según Wemple (21), hasta un valor de $\approx 3.2$. A partir del valor del parámetro de dispersión $E_{\mathrm{d}}=20.75 \mathrm{eV}$, correspondiente a la lámina vítrea de composición $\mathrm{As}_{33} \mathrm{~S}_{67}, N_{\mathrm{e}}=(33 \times 5+67 \times 6) / 67 \approx$ 8.5 y $Z_{\mathrm{a}}=2$, se obtiene un valor de $N_{\mathrm{c}} \approx 3.3$, que está en notable acuerdo con el deducido por Wemple.

La Ec. [11] fue aplicada satisfactoriamente por Tanaka (22), para explicar el aumento de $E_{\mathrm{d}}$ con el contenido de As en el sistema vítreo calcogenuro $\mathrm{As}_{\mathrm{x}} \mathrm{S}_{100-\mathrm{x}}$ (debido al aumento de la coordinación efectiva del As). Por lo tanto, es ciertamente razonable extender la validez de esta ecuación al caso que nos ocupa. Esto significaría, que la incorporación de Ag dentro de la matriz del material calcogenuro daría lugar a un aumento de uno o varios de los términos de la parte derecha de la Ec. [11]. Así, analizando dicha relación podríamos argumentar que, incluso si el dopaje con plata aumentara la contribución iónica de los enlaces, éste no puede ser, obviamente, el factor predominante en la tendencia encontrada en el parámetro de dispersión $E_{\mathrm{d}^{\prime}}$ ya que esto cambiaría $\beta$ hacia el valor iónico, con lo que $E_{\mathrm{d}}$ debería disminuir. Además, en materiales de mayor carácter iónico, la separación energética más acusada existente entre las bandas tipo "s" y las bandas tipo " $\mathrm{p}$ ", daría lugar a una disminución del término $N_{\mathrm{e}}$. Finalmente, dada la baja electronegatividad de la plata, ésta aparece como un catión en la estructura y, por tanto, el valor de $Z_{\mathrm{a}}=2$ seguiría siendo válido para todas las muestras.

Así pues, teniendo en cuenta los argumentos anteriores, es razonable asumir, entonces, que el factor dominante en el aumento encontrado para el parámetro de dispersión $E_{\mathrm{d}^{\prime}}$ debe ser un incremento del número de coordinación promedio de los cationes, $N_{c^{\prime}}$ debido a la incorporación de plata a la estructura. Esta idea se ve apoyada, además, por la alta coordinación que presenta la plata en ciertos compuestos cristalinos de Ag-As-S, con composiciones cercanas a las muestras fotodopadas con elevada concentración de plata. Por un lado, en la trechmannita, $\mathrm{AgAsS}_{2}(25 \%$ at. de $\mathrm{Ag}$ ), hay cuatro átomos de azufre a menos de $3 \AA$ de cada átomo de plata (23) (concretamente a distancias de 2.6, 2.6, 2.64 y $2.73 \AA$ ). Por otro lado, en la proustita $\mathrm{Ag}_{3} \mathrm{AsS}_{3}$ (42\% at. de Ag), la coordinación de la plata es tres (23) (en este caso los átomos de azufre se encuentran a distancias de $2.45,2.45$ y 2.79 Å). Así, en muestras fotodopadas con altas concentraciones de plata es de esperar que existan fragmentos cuya estructura sea muy similar a la del correspondiente compuesto cristalino, contribuyendo, por tanto, al aumento del valor promedio del número de coordinación. Esta contribución es de esperar que aumente con el contenido de plata, lo que explicaría la tendencia encontrada para el parámetro de dispersión, $E_{\mathrm{d}}$. Es necesario indicar que se ha encontrado plata altamente coordinada $\left(N_{\mathrm{Ag}}=4\right)$ en otros materiales calcogenuros amorfos que contienen dicho metal (24), lo que refuerza los argumentos expuestos.

En la Fig. 4 se muestra la dependencia del gap óptico, $E_{\mathrm{g}}{ }^{\mathrm{opt}}$, con el contenido de plata. El decrecimiento encontrado para este parámetro al aumentar el contenido de plata, es debido al hecho de que los enlaces Ag-S tienen una menor energía (217.1 $\left.\mathrm{kJ} \mathrm{mol}{ }^{-1}\right)$ que los enlaces As-S $\left(379.5 \mathrm{~kJ} \mathrm{~mol}^{-1}\right)$, lo que da lugar a una menor diferencia energética entre los bordes de movili- 
dad de las bandas de valencia y de conducción. En la Fig. 4 se representan además, los valores de $E_{\mathrm{g}}$ opt encontrados por Zakery et al. (25), para láminas de $\mathrm{As}_{30} \mathrm{~S}_{70}$ fotodopadas con plata, usando la técnica de incorporación de Ag en un "único paso". En este caso, aunque la lámina de partida tiene una composición ligeramente diferente a la nuestra, sus valores muestran un muy razonable acuerdo con los obtenidos en este trabajo. Finalmente, hemos comprobado que la relación entre el parámetro de dispersión, $E_{0}$ y el gap óptico, $E_{\mathrm{g}}$ opt, $E_{0} \approx$ $2 \times E_{\mathrm{g}}$ opt , encontrada por Tanaka (23) en láminas vítreas calcogenuras del sistema binario As-S, se verifica igualmente en láminas de composición química $\mathrm{Ag}_{\mathrm{x}}\left(\mathrm{As}_{0.33} \mathrm{~S}_{0.67}\right)_{100-x}$. En la Fig. 4 se muestra la representación de los valores del parámetro de dispersión $E_{0}$ obtenidos, frente a aquellos correspondientes al gap de Tauc, $E_{\mathrm{g}}$ opt para las láminas fotodopadas representativas con concentraciones de plata de $\mathrm{x}=3.0,8.6,13.6$ y $16.5 \%$ at., así como para la lámina inicial sin dopar. La dependencia lineal entre ambos parámetros es clara, siendo el valor de la pendiente de la recta de regresión 2.15.

\section{CONCLUSIONES.}

El método de caracterización óptica empleado en este trabajo para el cálculo del espesor y de las constantes ópticas de láminas vítreas calcogenuras fotodopadas con plata, de composición química $\mathrm{Ag}_{\mathrm{x}}\left(\mathrm{As}_{0.33} \mathrm{~S}_{0.67}\right)_{100-x^{\prime}}$ se ha aplicado satisfactoriamente a láminas de espesores iniciales comprendidos entre 800 y $1200 \mathrm{~nm}$. Dicho método permite determinar el espesor y el índice de refracción de las muestras con una precisión mejor que un $2 \%$. Las láminas fotodopadas fueron preparadas fotodisolviendo, sucesivamente, pequeñas capas de plata en el material calcogenuro. Hemos analizado la dispersión del índice de refracción, $n(\lambda)$, haciendo uso del modelo de WempleDiDomenico, basado en la aproximación del oscilador armónico simple. El aumento de la energía de dispersión, $E_{\mathrm{d}^{\prime}}$ con el contenido de plata, es atribuido a la presencia de átomos de plata con una alta coordinación en la matriz del material calcogenuro, incrementado, por tanto, la coordinación media del catión en la estructura vítrea. Asimismo, la incorporación de plata introduce nuevos enlaces Ag-S con menor energía que los enlaces As-S, lo cual explica el decrecimiento del gap ópti$\mathrm{co}, E_{\mathrm{g}}{ }^{\text {opt }}$, y del parámetro de dispersión, $E_{0}$, con el aumento del contenido de plata.

\section{AGRADECIMIENTOS}

Este trabajo ha sido parcialmente financiado por la Comisión Interministerial de Ciencia y Tecnología (Proyecto MAT980791) y por la Czech Grant Agency (Proyecto 203/99/0420). Los autores quisieran agradecer la ayuda prestada por D. José A. Angel en la realización de este trabajo.

\section{BIBLIOGRAFÍA}

1. P.J.S. Ewen y A.E. Owen, “High-performance glasses”, M. Cable and J.M. Parker Eds. (Blackie, London, 1992) p. 287-309.

2. R. Prieto-Alcón, E. Márquez, J.M. González-Leal, R. Jiménez-Garay, A.V. Kolobov y M. Frumar, "On the reversible athermal photo-vitrification of $\mathrm{As}_{50} \mathrm{Se}_{50}$ thin films deposited onto silicon wafer and glass substrates", Appl. Phys. A 68. 653661. (1999).

3. G. Dale, A.E. Owen y P.J.S. Ewen, “Metal-photodissolution in amorphous semiconductors of the As-S system", Physics and Applications of Non-Crystalline Semiconductors in Optoelectronics, A. Andriesh y M. Bertolotti Eds., NATO ASI Series (Kluwer Academic Publishers, Dordrecht, 1997) p. 45-60.

4. E. Márquez, A.M. Bernal-Oliva, J.M. González-Leal, R. Prieto-Alcón y R. JiménezGaray, "On the irreversible photo-bleaching phenomenon in obliquely-evaporated $\mathrm{GeS}_{2}$ glass films", J. Non-Cryst. Solids 222. 250-257. (1987).

5. I.Z. Indutnyi, A.V. Stronski, S.A. Kostioukevitch, P.F. Romanenko, P.E. Schepeljavi y I.I. Robur, "Holographic optical element fabrication using chalcogenide layers", Opt. Eng. 34. 1030-1039. (1995).

6. T. Arai, Y. Wakayama, H. Kudo, T. Kishimoto, J. Lee, T. Ogawa y S. Onari, “Ag photodoping in chalcogenide $\mathrm{As}_{2} \mathrm{~S}_{3}$ ", J. Non-Cryst. Solids 114. 40-42. (1989).

7. T. Wagner, M. Vlcek, V. Smrcka, P.J.S. Ewen y A.E. Owen, "Kinetics and reaction products of the photo-induced solid state chemical reaction between silver and amorphous $\mathrm{As}_{33} \mathrm{~S}_{67}$ layers", J. Non-Cryst. Solids 164-166. 1255-1258. (1993).

8. E. Márquez, R. Jiménez-Garay, A. Zakery, P.J.S. Ewen y A.E. Owen, “On the kinetics of $\mathrm{Ag}$ photodissolution in $\mathrm{As}_{2} \mathrm{~S}_{3}$ chalcogenide glass films: oscillatory behaviour of the reaction rate", Philos. Mag. B 63. 1169-1179. (1991).

9. R. Swanepoel, "Determination of the thickness and optical constants of amorphous silicon", J. Phys. E: Sci. Instrum. 16. 1214-1222. (1983).

10. P.J.S. Ewen, A. Zakey, A.P. Firth y A.E. Owen, “Compositional dependence of photodissolution kinetics in amorphous As-S films", J. Non-Cryst. Solids 97\&98 1127-1130. (1987).

11. J.S. Berkes, S.W. Ing Jr. y W.J. Hillegas, "Photodecomposition of amorphous $\mathrm{As}_{2} \mathrm{Se}_{3}$ and $\mathrm{As}_{2} \mathrm{~S}_{3}$ ", J. Appl. Phys. 42. 4908-4916. (1971).

12. J. Dikova, N. Starbov y K. Starbova, "The mechanism of photoinduced transformations in amorphous $\mathrm{As}_{2} \mathrm{~S}_{3}$ thin films", J. Non-Cryst. Solids 167. 50-58. (1994)

13. R. Prieto-Alcón, J.M. González-Leal, A.M. Bernal-Oliva y E. Márquez, “Photoamorphization and photo-oxidation of $\mathrm{As}_{50} \mathrm{Se}_{50}$ thin films deposited onto silicon substrates", Mater. Lett. 36. 157-161. (1998) .

14. R. Swanepoel, "Determination of the surface roughness and optical constants of inhomogeneous amorphous silicon films" , J. Phys. E: Sci. Instrum. 17. 896-903. (1984).

15. E. Márquez, J.B. Ramírez-Malo, P. Villares, R. Jiménez-Garay, P.J.S. Ewen y A.E. Owen, "Calculation of the thickness and optical constants of amorphous arsenic sulphide films from their transmission spectra", J. Phys. D: Appl. Phys. 25. 535541. (1992)

16. J.B. Ramírez-Malo, E. Márquez, P. Villares y R. Jiménez-Garay, “Refractive-index dispersion and optical-absorption edge of physically vapor-deposited films of the $\mathrm{As}_{35} \mathrm{~S}_{65}$ chalcogenide glass", Mater. Lett. 17. 327-332. (1993)

17. J. Tauc y A. Menth, "States in the gap", J. Non-Cryst. Solids, 8-10. 569-585. (1972).

18. M. McClain, A. Feldman, D. Kahamer y X. Ying, “An algorithm and computer program for the calculation of the envelope curves", Comput. Phys. 5. 45-48. (1990).

19. T. Kawaguchi y K. Masui, “Analysis of change in optical transmission spectra resulting from Ag photodoping in chalcogenide film", Jpn. J. Appl. Phys. 26. 15-21. (1987) .

20. S.H. Wemple y M. DiDomenico Jr., “Behavior of the electronic dielectric constant in covalent and ionic materials", Phys. Rev. B 3. 1338-1351. (1971) .

21. S.H. Wemple, "Refractive-index behavior of amorphous semiconductors and glasses", Phys. Rev. B 7. 3767-3777. (1973).

22. K. Tanaka, “Optical properties and photoinduced changes in amorphous As-S films", Thin Solid Films 66. 271-279. (1980).

23. Base de Datos ICSD (Inorganic Crystal Structure Database), FIZ Karlsruhe, GMELIN Institute Frankfurt y el Instituto de Química Inorgánica de la Universidad de Bonn (Alemania).

24. A. Fisher-Colbrie, A Bienenstock, P.H. Fuoss y M.A. Marcus, "Structure and bonding in photodiffused amorphous Ag-GeSe 2 thin films", Phys. Rev. B 38. 12388-12403. (1988).

25. A. Zakery, A. Zekak, P.J.S. Ewen, C.W. Slinger y A.E. Owen, “Optical constants of Ag-photodoped As-S amorphous films", J. Non-Cryst. Solids 114. 109-111. (1989). 